\title{
Functional Quality Criterion of Rock Handling Mechanization at Open-pit Mines
}

\author{
Yuri Voronov ${ }^{1 *}$, and Artyom Voronov ${ }^{1}$ \\ ${ }^{1}$ T.F. Gorbachev Kuzbass State Technical University, 650000, 28 Vesennyaya St., Kemerovo, Russia
}

\begin{abstract}
Overburden and mining operations at open-pit mines are performed mainly by powerful shovel-truck systems (STSs). One of the main problems of the STSs is a rather low level of their operating quality, mainly due to unjustified over-trucking. In this article, a functional criterion for assessing the quality of the STS operation at open-pit mines is formulated, derived and analyzed. We introduce the rationale and general principles for the functional criterion formation, its general form, as well as variations for various STS structures: a mixed truck fleet and a homogeneous shovel fleet, a mixed shovel fleet and a homogeneous truck fleet, mixed truck and shovel fleets. The possibility of assessing the quality of the STS operation is of great importance for identifying the main directions for improving their operational performance and operating quality, optimizing the main performance indicators by the quality criterion, and, as a result, for possible saving of material and technical resources for open-pit mining. Improvement of the quality of the STS operation also allows increasing the mining safety and decreasing the atmosphere pollution - by means of possible reducing of the number of the operating trucks.
\end{abstract}

\section{Introduction}

Since the quality of the shovel-truck system operation can only be manifested in the course of fulfilling its function in accordance with its purpose (timely excavation, loading and removal of rock) under certain conditions, the work of the STS as an unified system within the mine should be viewed as the result of interaction of its elements (mining shovels and trucks) under restrictions imposed by the external mining environment. The main purpose of assessing the quality of the interaction between shovels and trucks in the STS is to determine how effectively each of them performs its function under specific conditions. For such an assessment, it is necessary to have a functional criterion for joint work of shovels and trucks in the STS structure, taking into account constraints imposed by the external environment - including in terms of its protection and mining safety.

\section{Materials and methods}

\footnotetext{
*Corresponding author: ya.voronov454@yandex.ru
} 
A lot of scientific papers are devoted to the problem of determining the rational structure of the STS, for example [1-7]. In them we can single out one common point. From the point of joint work of mining shovels and trucks, the main purpose of shovels, which are leading machines of the system, is to ensure high productivity of the STS; the main purpose of trucks is the ability to ensure that this productivity is achieved, that is, the work of shovel and truck fleets should be balanced as much as possible.

It is possible to balance the total production of $N_{s}$ available shovels with the help of the necessary number of trucks $N_{t}$ by comparing the actual production of shovels, which are leading machines in the STS, with the potential production of the truck fleet.

The total shift operational performance (production) of the shovel fleet of the STS can be determined by the formula $\left(\mathrm{m}^{3} / \mathrm{shift}\right)$ :

$$
P_{\Sigma}^{s}=\frac{E_{b}^{s}}{t_{c}^{s}} \cdot N_{s} \cdot T_{s h} \cdot k_{O P}^{s}
$$

where $E_{b}^{s}$ - shovel bucket capacity, $\mathrm{m}^{3} ; t_{c}^{s}$-duration of the shovel cycle (time to load one bucket), h; $N_{s}$ - number of shovels in the STS; $T_{s h}$ - duration of the working shift, $\mathrm{h}$; $k_{O P}^{s}$ - operational performance ratio of the shovel fleet in the STS.

The operational performance ratio $k_{O P}^{s}$ is the ratio of the time of pure work of mining shovels to the duration of the shift, taking into account all regulated and unplanned downtimes. After transformations, it can be represented as:

$$
k_{O P}^{s}=1-\frac{\sum_{1}^{N_{s}} t_{D T}^{s}}{T_{s h} \cdot N_{s}}
$$

where $\sum_{1}^{N_{s}} t_{D T}^{s}$ - total downtime of $N_{s}$ shovels during the shift, $\mathrm{h}$.

The total theoretical productivity of the truck fleet $\left(N_{t}\right.$ units $)$ is $\left(\mathrm{m}^{3} / \mathrm{shift}\right)$ :

$$
P_{\Sigma}^{t}=\frac{E_{b}^{t}}{t_{c}^{t}} \cdot N_{t} \cdot T_{s h}
$$

where $E_{b}^{t}$ - truck body capacity, $\mathrm{m}^{3} ; t_{c}^{t}$ - truck cycle time from the end of previous load to the beginning of the next one, h; $N_{t}$ - number of trucks in the STS.

The ratio of these productivities $\left(P_{\Sigma}^{t}\right.$ and $P_{\Sigma}^{s}$ ) characterizes the balance of the joint work of shovel and truck fleets in the greatest degree, and, consequently, can serve as a functional criterion for assessing the quality of the STS operation. We have

$$
\lambda=\frac{P_{\Sigma}^{t}}{P_{\Sigma}^{s}}=\frac{E_{b}^{t} \cdot N_{t} \cdot T_{s h} \cdot t_{c}^{s}}{E_{b}^{s} \cdot N_{s} \cdot T_{s h} \cdot t_{c}^{t} \cdot k_{O P}^{s}} .
$$

Considering that $E_{b}^{t} / E_{b}^{s}=n_{b}$ is the number of buckets loaded into the truck body, and $t_{c}^{s} \cdot n_{b}=t_{l}^{s}$ is the time to load the truck by this shovel, after transformations we obtain: 


$$
\lambda=\frac{N_{t} \cdot t_{l}^{s}}{N_{s}-\frac{\sum_{1}^{N_{s}} t_{D T}^{s}}{T_{s h}} \cdot t_{c}^{t}} .
$$

In dependence (5), the value $\sum_{1}^{N_{s}} t_{D T}^{s} / T_{s h}$ is a part of the shovel fleet, which is not used during the shift due to downtimes. It can be designated as $N_{s}^{D T}$. Then we have

$$
N_{s}^{D T}=\frac{\sum_{1}^{N_{s}} t_{D T}^{s}}{T_{s h}}
$$

and

$$
\lambda=\frac{N_{t} \cdot t_{l}^{s}}{\left(N_{s}-N_{s}^{D T}\right) \cdot t_{c}^{t}} .
$$

Without taking into account the shovel downtime, dependence (7) degenerates into $N_{t} \cdot t_{c}^{s} / N_{s} \cdot t_{c}^{t}$, investigated in [8-12] and called "the match factor".

Actually, the functional criterion $\lambda$ (7) is the ratio of the rate of arrival of trucks for loading $\lambda_{t}$ and the rate of service them by shovels $\lambda_{s}$. If $\lambda=1$, then there is a certain equilibrium point, when trucks arrive to shovels with the same intensity with which they are serviced. If $\lambda>1$, trucks arrive faster than they are serviced, so we should expect the queues to appear. $\lambda<1$ shows that shovels operate faster than trucks arrive, and then downtime of shovels waiting for the truck arrival is likely to occur.

\section{Results and discussion}

Dependence (7) assumes that the arrival of trucks for loading is carried out evenly, that is, each subsequent truck arrives for loading exactly at the moment when the previous one leaves. However, it is almost impossible to achieve an even movement of trucks because of the large number of unforeseen factors. As a result, even if the number of trucks in the STS is quite enough to ensure the removal of all rock, which is loaded by available shovels during the shift, downtime of shovels and trucks will still occur because of the uneven arrival of trucks for loading. Great influence is exerted by the used strategy of truck allocation between shovels (with fixation for particular shovels, without fixation at all or according to a combined scheme). Besides the problem of improving truck loading is absent on the segments with direct dumping [13]. But this technology with internal dumping can be used on the quarry field with flat coal seams. And for inclined and steep seams using the excavator-and-truck complex is necessary. So there are many other aspects that are specific to a particular mine, for example: possibility of unforeseen interaction of trucks at the intersection points of mine roads, impossibility of overtaking, different quality of different road sections, poor crushing of rock during drilling and blasting (affects the loading time), different truck weariness and driver skills (affects the truck speed characteristics).

Dependence (7) also assumes that loading and transporting mining equipment fleets are homogeneous, that is, only one type (size) of trucks and shovels is used. However, in 
practice, there are mixed equipment fleets everywhere. Therefore, it makes sense to consider three options for joint operation of shovels and trucks in the STS: 1) a mixed fleet of trucks and a homogeneous fleet of shovels; 2) a mixed fleet of shovels and a homogeneous fleet of trucks; 3 ) both fleets are mixed.

1. Intensity of arrival of trucks for loading (auto/h):

$$
\lambda_{t}=\frac{N_{t}}{\overline{t_{c}^{t}}},
$$

where $\overline{t_{c}^{t}}$ - averaged cycle time (including idles) of all trucks during the whole shift, $\mathrm{h}$.

Obviously, loading time of a truck varies depending on the size of this truck. The rate of truck service by shovels $\lambda_{s_{i}}$ should reflect the time for servicing each $i$-th truck:

$$
\lambda_{s_{i}}=\frac{N_{s}-N_{s}^{D T}}{\overline{t_{l}^{s}}},
$$

where $\overline{t_{l}^{s}}$ - time to load the weighted average truck, $\mathrm{h}$.

Since the truck fleet is mixed, the value of $\overline{t_{l}^{s}}$ will be:

$$
\overline{t_{l}^{s}}=\frac{\sum_{1}^{N_{t}}\left(t_{l_{i}}^{s} \cdot N_{t_{i}}\right)}{N_{t}}
$$

where $t_{l_{i}}^{s}$ - time to load the truck of type $i, \mathrm{~h} ; N_{t_{i}}$ - number of trucks of type $i$ in the STS.

From dependences (9) and (10) we obtain:

$$
\lambda_{s_{i}}=\frac{\left(N_{s}-N_{s}^{D T}\right) \cdot N_{t}}{\sum_{1}^{N_{t}}\left(t_{l_{i}}^{s} \cdot N_{t_{i}}\right)}
$$

Since the functional criterion $\lambda$ is the ratio of the intensity of truck arrival to the intensity of shovel service, then dividing expression (8) by expression (11), we obtain:

$$
\lambda=\frac{\sum_{1}^{N_{t}}\left(t_{l_{i}}^{s} \cdot N_{t_{i}}\right)}{\left(N_{s}-N_{s}^{D T}\right) \cdot \overline{t_{c}^{t}}} .
$$

Despite the fact that the shovel fleet is homogeneous, the variance in the time to load different trucks by the same shovel leads to significant variances in the duration of truck cycles. Therefore, it makes sense to use unique values of cycle times for calculation of the functional criterion. This means that in formula (12), instead of the average cycle for all trucks, the weighted average value of unique truck cycles should be used. We have

$$
\overline{t_{c}^{t}}=\frac{\sum_{1}^{N_{t}}\left(t_{c_{i}}^{t} \cdot N_{t_{i}}\right)+\sum_{1}^{N_{t}} t_{D T_{i}}^{t}}{N_{t}}=\frac{\sum_{1}^{N_{t}}\left[\left(t_{c_{i}}^{t}+t_{D T_{i}}^{t}\right) \cdot N_{t_{i}}\right]}{N_{t}}
$$


where $t_{c_{i}}^{t}$-cycle time of the $i$-th truck without downtimes, h.

Substituting dependence (13) into expression (12), we obtain:

$$
\lambda=\frac{N_{t} \cdot \sum_{1}^{N_{t}}\left(t_{l_{i}}^{s} \cdot N_{t_{i}}\right)}{\left(N_{s}-N_{s}^{D T}\right) \cdot \sum_{1}^{N_{t}}\left[\left(t_{c_{i}}^{t}+t_{D T_{i}}^{t}\right) \cdot N_{t_{i}}\right]},
$$

where $N_{s}^{D T}$ is determined by dependence (6).

2. When using shovels of different sizes, the time taken to load identical trucks will vary. The exact definition of shovel service rate is achieved by introducing the least common multiple (LCM) for unique values of the loading time in each "truck-shovel" pair. Calculating how many loading cycles is contained in the LCM, we can find the number of trucks serviced in a given period of time. Then the intensity of service of trucks by shovels of different sizes will be determined as

$$
\lambda_{s_{j}}=\frac{\sum_{1}^{N_{s}}\left[\left(N_{s_{j}}-N_{s_{j}}^{D T}\right) \cdot \frac{Z_{t_{l}}}{t_{l_{j}}^{s}}\right]}{Z_{t_{l}}},
$$

where $N_{s_{j}}$ - number of shovels of type $j$ in the STS; $N_{s_{j}}^{D T}$ - number of idling shovels of type $j ; Z_{t_{l}}-$ LCM for individual loading times for all $j$-s; $t_{l_{j}}^{s}$ - time for shovel of type $j$ to load the truck of the only type, $h$.

Considering that the functional criterion $\lambda$ is the ratio of the intensity of arrival of trucks (8) to the intensity of their service (15), we obtain:

$$
\lambda=\frac{N_{t} \cdot Z_{t_{l}}}{\overline{t_{c}^{t}} \cdot \sum_{1}^{N_{s}}\left[\left(N_{s_{j}}-N_{s_{j}}^{D T}\right) \cdot \frac{Z_{t_{l}}}{t_{l_{j}}^{s}}\right]} .
$$

If there are several dumps, we can extend equation (16) for different truck cycles:

$$
\lambda=\frac{N_{t}^{2} \cdot Z_{t_{l}}}{\sum_{1}^{N_{s}}\left[\left(N_{s_{j}}-N_{s_{j}}^{D T}\right) \cdot \frac{Z_{t_{l}}}{t_{l_{j}}^{s}}\right] \cdot \sum_{1}^{N_{t}}\left[\left(t_{c_{i}}^{t}+t_{D T_{i}}^{t}\right) \cdot N_{t_{i}}\right]},
$$

where $N_{s_{j}}^{D T}$ is determined by dependence (6).

3. In the case of mixed truck and shovel fleets, it is necessary to first determine the time required for each shovel to service the available fleet of trucks. The loading and idle time ( $t_{L_{j}}$ ) for each $j$-th type of shovels is defined as

$$
t_{L_{j}}=\sum_{1}^{N_{t}}\left(t_{l_{i j}}^{s} \cdot N_{t_{i}}\right)
$$


где $t_{l_{i j}}^{s}$ - time for shovel of type $j$ to load the truck of type $i, \mathrm{~h}$.

Then the intensity of service of all trucks by all shovels is

$$
\lambda_{s}=\sum_{1}^{N_{s}}\left(\frac{N_{s_{j}}-N_{s_{j}}^{D T}}{t_{L_{j}}}\right) \cdot N_{t} .
$$

If the truck cycle time is taken as the average over the entire fleet for a given period, then the functional criterion is defined as

$$
\lambda=\frac{1}{\sum_{1}^{N_{s}}\left(\frac{N_{s_{j}}-N_{s_{j}}^{D T}}{t_{L_{j}}}\right) \cdot t_{c}^{t}} .
$$

In the case of unique truck cycles, this formula can easily be extended to this:

$$
\lambda=\frac{N_{t}}{\sum_{1}^{N_{s}}\left[\frac{N_{s_{j}}-N_{s_{j}}^{D T}}{\sum_{1}^{N_{t}}\left(t_{l_{i j}}^{s} \cdot N_{t_{i}}\right)}\right] \cdot \sum_{1}^{N_{t}}\left[\left(t_{c_{i}}^{t}+t_{D T_{i}}^{t}\right) \cdot N_{t_{i}}\right]} .
$$

In the dependence (21), four unknown values are fundamental: $t_{l_{i j}}^{s}, t_{c_{i}}^{t}$ as well as $\sum_{1}^{N_{t}} t_{D T_{i}}^{t}$ and $\sum_{1}^{N_{s}} t_{D T_{j}}^{s}$ (downtime of trucks and shovels waiting for the work, respectively).

The values of $t_{l_{i j}}^{s}$ and $t_{c_{i}}^{t}$ can be determined analytically or taken according to the factual data of the mine. And if it is possible to determine $t_{l_{i j}}^{s}$ analytically quite accurately, then the truck cycle time $t_{c_{i}}^{t}$ is difficult. Therefore, in order to avoid incorrect results, it is desirable to use actual values, especially since automated fleet management systems operating at most mines are able to record the time of all operations to within a second. Analytical methods should be used if such a system is not installed on mining trucks.

Total downtimes $\sum_{1}^{N_{t}} t_{D T_{i}}^{t}$ and $\sum_{1}^{N_{s}} t_{D T_{j}}^{s}$ are purely stochastic values, therefore their analytical determination is impossible. They should be taken according to the mine data.

\section{Conclusion}

A distinctive feature of the functional criterion for a system of machines (STS) is the provision of rational interaction of its component mining shovels and trucks. Shovels as the leading machines of the system should provide the highest possible productivity of the STS, the task of the trucks is to ensure the achievement of this productivity, that is, the work of the shovel and truck fleets should be balanced as much as possible.

The degree of balance of the total productivity of the shovel fleet at a particular mine with the help of a truck fleet, which can be used for this, can be estimated by comparing the intensity of arrival of trucks for loading and servicing them by shovels. Three variants of 
the STS structure are considered, for each of which specific formulas for calculating the functional criterion were obtained.

As practice shows, the number of simultaneously operating trucks at existing mines is usually overestimated. Formation of the STS with the help of the proposed functional criterion will allow to reduce the number of trucks at the mine. This will reduce the probability of collisions, as well as the level of harmful emissions, thus increasing the safety and environmental friendliness of mining operations.

\section{References}

1. G.H. Blackwell, CIM Bulletin, 92:1028, 143 (1999)

2. C.H. Ta, J.V. Kresta, J.F. Forbes, H.J. Marquez, International Journal of Surface Mining, Reclamation and Environment, 19:3, 162 (2005)

3. A. Krause, C. Musingwini, The Journal of The Southern African Institute of Mining and Metallurgy, 10, 469 (2007)

4. S.G. Ercelebi, A. Bascetin, The Journal of The Southern African Institute of Mining and Metallurgy, 109, 433 (2009)

5. M.A. May, Applications of queuing theory for open-pit truck-shovel haulage systems, 79 (MSc thesis, Virginia Polytechnic Institute and State University, USA, 2012)

6. C. Burt, L. Caccetta, Interfaces, 44:2, 143 (2014)

7. S. A. Dindarloo, M. Osanloo, S. Frimpong, The Journal of The Southern African Institute of Mining and Metallurgy, 115, 209 (2015)

8. J. Douglas, Prediction of shovel-truck production: a reconciliation of computer and conventional estimates (Department of Civil Engineering, Stanford University, USA, 1964)

9. W. Morgan, L. Peterson, Mining Engineering, 76 (1968)

10. C. Burt, An optimisation approach to materials handling in surface mines, 168 (Curtin University of Technology, Australia, 2008)

11. C. Burt, L. Caccetta, P. Welgama, L. Fouche, Journal of the Operational Research Society, 62:8, 1498 (2011)

12. S. Nel, M.S. Kizil, P. Knights, 35th APCOM Symposium, Wollongong NSW, 381 (2011)

13. M.A. Tyulenev, T.N. Gvozdkova, S.A. Zhironkin, E.A. Garina, Geotech. Geol. Eng., 35:1, 203-212 (2017) 
